ORIGINAL ARTICLE

\title{
Health Seeking Behavior of Patients Diagnosed with Cervical Cancer in Addis Ababa, Ethiopia
}

\author{
Zinaw Mesafint $^{1 *}$, Yemane Berhane ${ }^{2}$, Dawit Desalegn ${ }^{3}$
}

\footnotetext{
OPEN ACCESS

Citation: Zinaw Mesafint, Yemane Berhane, Dawit Desalegn. Health Seeking Behavior of Patients Diagnosed with Cervical Cancer in Addis Ababa, Ethiopia. Ethiop J Sci.2018;28(2):111. doi:http://dx.doi.org/10.4314/ejhs.v28i2.2. Received: August 29, 2017

Accepted: September 8, 2017

Published: March 1, 2018

Copyright: (c) 2018 Zinaw Mesafint., et al . This is an open access article distributed under the terms of the Creative Commons Attribution License, which permits unrestricted use, distribution, and reproduction in any medium, provided the original author and source are credited. Funding: Nil

Competing Interests: The authors declare that this manuscript was approved by all authors in its form and that no competing interest exists.

Affiliation and Correspondence:

${ }^{1}$ Tikur Anbessa Specialized Hospital,

Addis Ababa University

${ }^{2}$ Addis Continental Institute of Public Health

*Email: zinawdessie@gmail.com
}

\begin{abstract}
BACKGROUND: Cervical cancer is increasingly recognized as one of the public health problems among women in developing countries. Most women with cervical cancer are seen in the health care system late with advanced stage of cancer. This study aims to explore the care seeking behavior of women with cervical cancer.

METHODS: A qualitative study was conducted to explore the health seeking behavior of women with cervical cancer for patients who were admitted with cervical cancer in Tikur Anbessa Hospital. In-depth interviews were conducted with purposively selected participants. Interviews were transcribed verbatim and then translated into English and analyzed using a content analysis approach. The study period was from October 2013 to April 2014. RESULTS: The study found that women diagnosed with cervical cancer lack prior awareness about cervical cancer and availability of screening services. Moreover, decision making to seek medical care up on experiencing early symptoms seems to be delayed. Women reported the first symptom was unusual vaginal bleeding that forces them to seek care. The bleeding could be profound and accompanied by offensive odor. Fear of stigmatization could make women reluctant to seek timely care. Once treatment is started most women found it shockingly expensive.

CONCLUSION: Women lack general awareness about the nature and symptoms of cervical cancer. All health services providers serving women need to sensitize women to seek timely screening services and timely treatment services if cervical cancer is confirmed.

KEYWORDS: Cervical Cancer, Health Seeking Behavior, Cervical Screening
\end{abstract}




\section{INTRODUCTION}

Cervical cancer is among the common reproductive health disease and still continues to be the major cause of death of women across the world in their later life (1). It is a leading cause of women in developing countries where financial and social stability is not well secured (2). Cervical cancer has been associated with sexually transmitted infections particularly with the Human Papilloma virus

which is sexually transmitted and believed to be the main cause of carcinogen in humans (3).

The developing countries where sexually transmitted infections are rampant account for more than $75 \%$ of the annual number of cervical cancer cases that occur worldwide $(4,5)$. Studies suggested that most cancer patients in subSaharan Africa tend to present themselves for health care with advanced stage of the disease $(6,7,8)$.

Where HIV prevalence is high, among the reproductive age women, the risk of acquiring Human Papilloma Virus infection has been high (9). Cervical carcinoma was found to be the most prevalent malignant disease in hospitalized adult females (10). However, despite increasing recognition of cervical carcinoma, only few women receive screening services (11).

Due to low level of awareness and lack of adequate screening services, most women with cervical cancer are diagnosed at advanced stage hence resulting in poor prognosis (12). Thus, it is imperative to conduct studies to understand the facilitators and barriers of early screening/diagnosis of cervical cancer in Ethiopia.

Although the number of cervical cancer cases being seen in Ethiopian health facilities is increasing, the health seeking behavior of those patients has not been systematically studied. Thus, this study was conducted to explore the health care seeking behavior of women diagnosed with cervical cancer.

\section{MATERIALS AND METHODS}

TikurAnbessa is the only referral hospital with radiation and surgical oncology services in the country so far and adequate number of samples assumed to be obtained in Gynecology and Oncology units. The hospital provides a variety of specialty and sub-specialty services but specifically departments of Gynecology/Obstetrics OPD, and Oncology wards were selected to be the study areas. In Addition, the Addis Ababa City Cancer Registry is placed in this hospital.

A qualitative study was carried out to gather relevant information to address the objectives of the study. Purposively selected women followed by criterion sampling who were diagnosed with cervical cancer suggested by histological findings and/ or receiving treatment at the time of the study participated in in-depth interviews. A total of 12 women participated in the study. The study period was from October 2013-April 2014, and exist interviews were conducted in a private space in the hospital and at a time most convenient for the study participants. The average length of time for the interview was about 40 minutes.

Interviews were conducted using semistructured interview guide. All interviews were audio-recorded with the consent of the participants. All interviews were conducted by the principal investigator in Amharic language, and it was transcribed and then translated into English.

A content analysis was done using Open Code 4.0 computer software which helps to systematically code and categorizes qualitative data. Ethical clearance for the study was obtained from Haramaya University 


\section{RESULTS}

The study population included patients diagnosed with cervical cancer at Gynecology OPD, Oncology, and Gynecology wards of TikurAnbessa Hospital. Study participants came from different regions of Ethiopia: Amhara and Oromia regions, Gurage Zone, and Dire Dawa and Addis Ababa cities. Participants' age ranged from 25-67 years.

The findings are presented in seven thematic areas, and the themes were developed and categorized after the responses of the participants. This method of category is based on the Health Belief Model (HBM) which serves as a useful method of understanding participants' perception and beliefs specifically in utilizing existing services.

Awareness of cervical cancer: Almost all participants had very low knowledge and awareness about cervical cancer before they were diagnosed. A patient from Northern Amhara region said ..."Initially, I came to Addis Ababa to attend Nephew's Christening, I told my brother and his wife about my bleeding and decided to use the opportunity to have it checked. Then, first they took me to Zewditu Memorial Hospital where I got examined and referred to TikurAnbessa Hospital for treatment. Otherwise, I would have gone home as soon as the Christening is over as I didn't realize I would be seriously ill [a 42-year-old woman on chemotherapy].

Another, a 50 year-old woman in the Oncology ward, said.... "for two years I neither had my periods nor pregnancy then I suddenly began to observe vaginal bleeding and went to see a doctor. They took tissue sample from my cervix for further examination which confirmed the diagnosis. Then, I was referred to Tikur Anbessa Hospital where after repeated appointments. I was finally admitted to Oncology ward and received radiation treatment. My condition improved, but only for a while as my cervix became infected again and now I am taking antibiotics and that is how I knew what cervical cancer is".

Perceived etiology: Most respondents associated their cause of illness to either repeated abortion they had, punishment from God, use of intra uterine device or to hard labor work they did. One of the respondents said the following about the cause of cervical cancer ... "I had intra-uterine device that stayed longer and that was the cause for my disease" [a 65 year-old woman in the Oncology ward].

Another woman from the Oncology ward said ..." I believe the cause is hard labor work. I used to work in a tannery company which was a hard job. I am retired from the factory, and I am now working in dry cleaning which is still a hard job".

Perceived symptoms and signs of the disease: Most of the respondents mentioned that vaginal bleeding is one of the dominant symptoms of cervical cancer. Some of them also mentioned back pain, itching, swelling around vaginal area and lower leg pains. The majority of the respondents explained that the extent of vaginal bleeding was massive and very difficult to tolerate. The major reason for seeking care has not been understanding the severity or the consequences of the illness but the intolerable bleeding.

One of the respondents said, "I used to change pad 5-6 times per day to contain the bleeding, which is very costly, and wash it frequently because the pad with massive bleeding is very irritant. I used to spend most of my time at home in order to get access to washing facility" [a 50 year- old woman in the Oncology ward].

Another woman expressed the extent of vaginal bleeding and associated discomfort as follows "...the bleeding was profuse and continuous; I wash it, wash it, and wash it. It was non-stop. I couldn't even sit on a chair properly. I used to put additional pads but that was not enough and affordable. I also used cloth instead of pad to minimize cost but that was very uncomfortable....oh! I suffered a lot".

Perceived benefit of cervical screening: The majority of the respondents were not aware of existing screening services for cervical cancer. Only a few knew about the availability of screening services and actually used it. However, even those who used the screening services did not know how frequently it should be done and rather they questioned the reliability of the screening test "Yes, I have under gone Pap smear test a long time ago and was told the 
test result was negative. The latest screening test I did in a private hospital was positive. If I was properly investigated and referred to another institution at an earlier stage, my disease wouldn't have advanced to this stage. They could have referred me directly to Tikur Anbessa Hospital" [a 46 year-old woman at OPD].

Perceived benefit of treatment: Modern therapy is perceived to be beneficial by most of the respondents. None of the participants attempted to be treated with traditional therapy except two of the participants who tried to be treated with holly water (blessed water)

A 46 year-old participant said that there are different treatment options women could seek in the absence of access to modern therap: "I tried holly water but it didn't work. However, you can't say God didn't cure me, you simply say, it was not the right remedy for me. Now, I came to try this modern therapy".

Health seeking behavior: Almost all of the respondents were either advised or financed by their families and friends to seek care. A 28 year-old woman on chemotherapy said, " A friend advised me initially to go to Family Guidance Association of Ethiopia for checkup as she was attending her follow-up with the same disease at Zewditu Memorial Hospital. Cost of medicines is my challenge, however, bed is free because I brought a support letter from 'kebele' that suggests I am poor ".

Another woman on chemotherapy said, "My relative here in Addis Ababa decided I should get treatment and the cost is covered by my brothers and daughters".

Challenges experienced: Apart from financial cost they incurred, most of the interviewees put vaginal bleeding at the top of their challenges associated with stigma. Some of them explained that vaginal bleeding at later stage changes to grey in color (yezengadawuha) with very unpleasant and offensive odor, which forces them to stay away even from their families.

A rural woman said, "... there is a river near my home and I used to go there and wash every time I have bleeding. I always do the ritual early in the morning so, my sons wouldn't see me. But, I lost a lot of blood without being aware of it. When I visited a private hospital for another reason, my blood tests showed that I have lost a lot of blood. The doctor urgently ordered blood transfusion, and I received five units of blood" [a 65 year-old woman in the Oncology ward].

Another woman on chemotherapy said, "I had profuse vaginal bleeding which later changed into grey color and became unpleasant and offensive. When people came to visit me, I feel ashamed because I thought it smells for them as well".

\section{DISCUSSION}

The study found that there is a considerable lack of awareness among participants about cervical cancer, and they seek care mostly when they experience intolerable bleeding. Thus, most seek care at a very advanced stage of the cervical cancer. The associated shame with advanced stage cancer that produces offensive smell and fear of the perceived cost of medical care were found to be barriers to seeking care even when the woman felt medical care is needed. Most women thus were persuaded by relatives and friends to seek care. The perceived cause of the cancer include repeated history of abortion, engaging in hard labor-intensive work for a long period of time and using intrauterine devices. Most respondents preferred modern treatment to tradional treatment to their illness. Previous studies indicated that the cost of treatment is the major barrier to seeking treatment among cancer patients (15). Women opt for traditional therapy to modern treatment due to costtraditional services provided for cancer care cheaper compared to modern cancer therapy (10). However, most of our participants preferred using modern therapy to traditional therapy as reported elsewhere (13). Most participants were not aware of the routine periodic need for cervical cancer screening after a certain age. The lack of awareness has prevented them to use available services. Some may even have visited health facilities for other conditions but not given the chance to do such tests which indicates the lack of awareness or readiness of health care providers to offer advice for women who might need cervical screening $(3,12)$. 
Most participants did not decide by themselves to seek medical care. They are either advised or financed by families or friends to seek medical treatment. This kind of challenge is common in low income countries. As the treatment of cancer is generally considered and actually is very expensive and prolonged, timely seeking of care even after becoming aware of the condition could be delayed. Thus, concerned bodies need to find a solution to this formidable barrier to seeking care for women in low-income countries. The challenge is going to grow as the life expectancy improves and the number of women requiring cancer treatment increases (4).

It is also time to think expanding facilities for screening and testing in and outside of the capital city, Addis Ababa, in order to identify cervical cancer at its earlier stage (14). This study shows the lack of cancer awareness among women, and healthcare providers operating outside referral centers. Thus, efforts to create cervical cancer awareness need to be strengthened.

\section{REFERENCES}

1. Proma paul, Jenifer L. winkler, Rosario M., Bartolini, Mary E.penny, Trinh Thu Huong and etal. Screen and treat approach to Cervical Cancer prevention using Visual Inspection with Acetic Acid and cryotherapy: experiences, perceptions and beliefs from demonstration projects in Uganda, Vietnam and Peru. The Onchologist. 2013;18:1278-1284.

2. Alemayehu Hailu and DamenHailemariam. Patient Side cost and its predictors for cervical cancer in Ethiopia. Biomed central. 2013;13:69.

3. R Smita Prasad. Management Strategies and Cost effectiveness in the prevalence of cervical cancer. Dove press Journal. 2009; 1 17-23.

4. Crispin Kahesa, Susanne Kjaer, Julius Mwaiselag, TwalibNgoma, Britt Tersbol, Myassa, Dartell and VibekaRasch. Determinants of acceptance of cervical cancer screening in Dare Selam. Biomed central. 2012; 12: 1093.
5. Hyacinth I, Hyacinth, Oluwatoyosi A Adekeye, Joy N. Ibeh, JolulopeOsoba. Cervical cancer and PAP smear awareness and Utilization of PAP smear test among Federal Civil servants in North Central Nigeria. Plose one Journal. 2012;10:1371.

6. Mwaka A, Okelo E, Kiguli J and Rutebemberwa E. An exploration of lay perceptions, beliefs and knowledge about cervical cancer among the Acholi in northern Uganda. BMC. 2014; 14726874/14/84.

7. Danielle N. Poole, J. Kathleen Tracy, Lauren levitz, maliRochas, KotouSangare, ShahlaYekta et al. A cross sectional study to assess HPV knowledge and HPV vaccine acceptability in Mali. PLOSONE.2013;8(2):page 2.

8. Kantelhardt Eva,Moelle U, begoihn M, Addissie A, Trocchi P, Yonas B, and et al. Survival of 1,059 patients who received Onchology therapy, Ethiopia. The onchologist. 2014;19: 727-734.

9. Mingo A, Panozzo C, Diangi Y, Smith J, steenhoff $\mathrm{A}$, Brewer $\mathrm{N}$ and et al. Cervical cancer awareness and screening in Botewana. HHS. 2012; 22(4): 638-644.

10. Mona M Rashed Alemayehu Bekele. The prevalence and pattern of HPV-16 immunostaining in uterine cervical carcinomas in Ethiopian women. Pan African medical Journal. 2010; 8:21.

11. Gedefaw A, Astatkie A, Tessema G, Ethiopia. The prevalence of precancerous Cervical cancer lesion among HIV infected women in southern Ethiopia. PLOS ONE. 2013; e84519.diol 10.1371.

12. Frehiwot Getahun, FekaduMazengia, MuluneshAbuhay and Zelalem Birhanu. Comprehensive knowledge about Cervical cancer is low among women in North West Ethiopia. Biomed central.2013; 13:2.

13. Mariam Faroqui, Mohamed A Hassali, Aishah K shatar, asrul A Shafie, Jan B Seang and Mohamed A Farooqui. A qualitative exploration of Malaysian cancer patients'perspectives on cancer and its treatment,Malaysia. Biomed central. 2011;11:525. 
14. Victoria K Fort, Mary sue Makin, Aaron J siegler, Kevin Ault, Roger Rochat .Barriers to cervical cancer screening in Mulanje. Dove press Journal. 2011;5: page 125.

15. Savard MH, Savard J, Quesnel C, Ivers H: The Influence of Breast Cancer, treatment on the Occurrence of Hot Flashes. Journal of Pain and Symptom Management 2009, 37(4):687-697. 